\title{
PARTLY TRANSITIVE MODULES AND MODULES WITH PROPER ISOMORPHIC SUBMODULES
}

\author{
BY \\ R. A. BEAUMONT AND R. S. PIERCE
}

I. Introduction. In this paper the problem of determining all $R$-modules $M$ over a principal ideal domain $R$ which have proper isomorphic submodules is considered. Such modules are called $I$-modules. In the generalization to modules from ordinary abelian groups, it is only in questions involving cardinality that there is any change, for the cardinality of the group is replaced by the cardinality of a set of generators. Abelian groups with isomorphic proper subgroups have been considered by Baer [1] and Beaumont [2]. For completeness, we restate in the present context some elementary lemmas which appear in [2], and we use Baer's theorem [1, Theorem 3] to prove other fundamental results, as well as in the proofs of Theorem 1 and Theorem 4, both of which are generalizations of a result of Kaplansky [4]. We adopt throughout the terminology and notation of Kaplansky's monograph [5].

Standard techniques from the theory of abelian groups are used to show that divisible and reduced modules may be considered separately, and to dispose of the divisible case. Necessary and sufficient conditions are given for a module $M$ to be an $I$-module when $M$ is divisible (Theorem 2), and when $M$ is a module of finite rank (Theorem 4). A torsion module of finite rank is not an $I$-module (Theorem 1 ), and a torsion-free module which is not divisible is an $I$-module (Theorem 3 ). For reduced p-primary modules (which is the only case left to be considered when $M$ is a torsion module), we use a method of Fuchs [3] to show that if the cardinality of a minimal set of generators of $M$ is greater than $\|R\| \aleph_{0}$, where $\|R\|$ is the cardinality of $R$, then $M$ is an $I$-module (Theorem 5 ). The same result holds, if the cardinality of a minimal generating set is $\boldsymbol{\aleph}_{0}$ (Theorem 8 ). This final result is based on the concept of a partly transitive module which is introduced in III, and on the result (Theorem 7 ), that countably generated $p$-primary modules are partly transitive.

For $p$-primary reduced abelian groups $G$ our results show that $G$ has isomorphic proper subgroups if the cardinality of $G$ is $\boldsymbol{\aleph}_{0}$ or is greater than $2 \boldsymbol{\aleph}_{0}$.

We have the following basic definitions:

Definition 1 . An $R$-module $M$ over a principal ideal domain $R$ is called an $I$-module if $M$ contains a proper isomorphic submodule.

Definition 2. A submodule $N$ of $M$ is called partly invariant if every isomorphism of $M$ into itself carries $N$ into itself.

Fully invariant submodules are partly invariant and partly invariant sub-

Presented to the Society June 15, 1957; received by the editors July 1, 1957. 
modules are characteristic. Suitable examples show that there exist modules for which these classes of submodules are distinct. Partly invariant submodules are the subject of another paper by the present authors.

The following results are from [1] and [2]:

BAer's Theorem: The $R$-module $M$ is not an I-module if there exists a well-ordered ascending chain of partly invariant submodules $M(\alpha)$ of $M$ with the following properties:

(i) $M(0)=0$ and $M(\gamma)=M$ for some ordinal $\gamma$.

(ii) $M(\alpha+1) / M(\alpha)$ is not an I-module.

(iii) If $\beta$ is a limit ordinal, then every element of $M(\beta)$ is contained in some $M(\alpha)$ for $\alpha<\beta$.

Lemma 1. If $M$ has a direct summand which is an I-module, then $M$ is an I-module.

LEMмA 2. If $M$ is the direct sum of infinitely many copies of the same $R$ module, then $M$ is an I-module.

II. I-modules. In this section, we prove some additional basic lemmas, and proceed with the classification of $I$-modules.

Lemma 3. If $M=M_{1} \oplus M_{2}$, where $M_{1}$ is a partly invariant submodule of $M$, then $M$ is an I-module if and only if either $M_{1}$ is an I-module or $M_{2}$ is an $I$ module.

Proof. If either $M_{1}$ or $M_{2}$ is an $I$-module, then $M$ is an $I$-module by Lemma 1. Conversely, $0, M_{1}, M_{1} \oplus M_{2}$ is an ascending chain of partly invariant submodules which satisfy the conditions of Baer's Theorem if neither $M_{1}$ nor $M_{2}$ is an $I$-module.

LemmA 4. If $M=\sum_{\beta<\gamma} \oplus M_{\beta}$, where each $M_{\beta}$ is a partly invariant submodule of $M$, then $M$ is an I-module if and only if at least one $M_{\beta}$ is an $I$ module.

Proof. The sufficiency follows from Lemma 1. Conversely, the submodules $M(\alpha)=\sum_{\beta<\alpha} \oplus M_{\beta}$ form an ascending chain of partly invariant submodules which satisfy the conditions of Baer's Theorem if each $M_{\beta}$ is not an $I$-module.

Lemma 5. A finitely generated torsion module $M$ is not an I-module.

Proof. If $M$ is finitely generated, each $p$-primary component $M_{p}$ in the primary decomposition of $M$ is finitely generated. Since each $M_{p}$ is fully invariant, it follows from Lemma 4 that we may suppose that $M$ itself is $p$ primary for some prime $p \in R$.

If $M$ is finitely generated and primary, $M=\sum_{i=1}^{k} \oplus\left\{x_{i}\right\}$, where $\left\{x_{i}\right\}$ is a cyclic module of order $p^{k_{i}}$. Let $M(j)=\left\{x \in M \mid p^{i} x=0\right\}$. Then $M(j+i) / M(j)$ is a direct sum of a finite number of cyclic modules of order $p$ and may be 
regarded as a finite dimensional vector space over the field $R /(p)$. Moreover any $R$-isomorphism of $M(j+1) / M(j)$ into itself is an $R /(p)$-isomorphism. Thus $M(j+1) / M(j)$ is not an $I$-module, since being finite dimensional, it does not contain an isomorphic proper subspace. With $M=M(\omega)$, the ascending chain of submodules $M(j)$ satisfy the conditions of Baer's Theorem.

We obtain an improvement of Kaplansky's Theorem in [4]. A module $M$ is of finite rank $r>0$ if every finite subset of $M$ not consisting of 0 alone is contained in a submodule generated by $r$ elements, and $r$ is the least positive integer with this property. If no such $r$ exists, then $M$ is said to have infinite rank (see [4] or [5] p. 49).

Theorem 1. A torsion module $M$ of finite rank is not an I-module.

Proof. If $M$ has finite rank, then each of its primary components has finite rank, so that by Lemma 4 we may suppose that $M$ is primary. With $M$ primary of finite rank $r$, by a result proved first by Prufer [7], $M$ is the direct sum of $r$ modules each cyclic or of type $p^{\infty}$. Since the set of elements in a module of type $p^{\infty}$ with orders dividing $p^{j}$ is a cyclic submodule of order $p^{j}$, the submodule $M(j)=\left\{x \in M \mid p^{i} x=0\right\}$ is finitely generated. Thus, for each $j$, $M(j+1) / M(j)$ is finitely generated, and is not an $I$-module by Lemma 5 . With $M=M(\omega)$, the ascending chain of submodules $M(j)$ satisfy the conditions of Baer's Theorem, to complete the proof of the theorem.

We now consider an arbitrary $R$-module $M$ and apply Lemma 3 to the decomposition $M=M_{1} \oplus M_{2}$, where $M_{1}$ is the maximal divisible submodule of $M$ and $M_{2}$ is reduced. Since $M_{1}$ is fully invariant, the hypothesis of Lemma 3 is satisfied. Thus we may consider divisible and reduced modules separately.

If $M$ is divisible, $M=T \oplus F$, where $T$, the torsion submodule of $M$, is a direct sum of modules of type $p^{\infty}$ for various primes $p \in R$, and $F$, which is torsion-free, is a direct sum of copies of $K$, the quotient field of $R$. Since $T$ is fully invariant, this decomposition satisfies the hypothesis of Lemma 3, and we may consider torsion-free and torsion divisible modules separately. The following lemmas are closely related to certain results of [2].

Lemma 6. A torsion-free divisible $R$-module $M$ is an $I$-module if and only if $M$ is a direct sum of infinitely many copies of $K$, the quotient field of $R$.

Proof. $M$ may be regarded as a vector space over $K$, and a module isomorphism of $M$ into itself is a nonsingular linear transformation of $M$.

Lemma 7. A torsion divisible R-module $M$ is an I-module if and only if at least one of the primary components of $M$ is a direct sum of infinitely many modules of type $p^{\infty}$.

Proof. If $M$ is an $I$-module, then at least one of its primary components is an $I$-module by Lemma 4 . Such a primary component cannot be of finite rank by Theorem 1 , hence must be the direct sum of infinitely many modules of type $p^{\infty}$. The converse follows from Lemmas 1 and 2 . 
The following theorem disposes of the divisible case.

Theorem 2. Let $M$ be a divisible R-module. Then $M$ is an I-module if and only if there exists a primary component $T_{p_{j}}$ of the torsion submodule $T$ of $M$ such that $M / \sum_{i \neq j} \oplus T_{p_{i}}$ has infinite rank.

Proof. Now $M=\sum_{i} \oplus T_{p_{i}} \oplus F$, where $F$ is torsion-free, and $M / \sum_{i \neq j} \oplus T_{p_{i}}$ $\cong T_{p_{j}} \oplus F$. If $M / \sum_{i \neq j} \oplus T_{p_{i}}$ has infinite rank then either $T_{p_{j}}$ or $F$ has infinite rank [7, p. 39]. If $F$ has infinite rank, then $F$ is the direct sum of infinitely many copies of $K$, and $M$ is an $I$-module by Lemma 1 and Lemma 6 . If $T_{p_{j}}$ has infinite rank, then $T_{p_{j}}$ is the direct sum of infinitely many modules of type $p^{\infty}$, and again $M$ is an $I$-module by Lemma 1 and Lemma 7 .

Conversely, if $M / \sum_{i \neq j} \oplus T_{p_{i}}$ has finite rank for every primary component $T_{p_{j}}$ of $T$, then $T_{p_{j}} \oplus F$ has finite rank for every $j$. Thus $F$ has finite rank and $T_{p_{j}}$ has finite rank for every $j$. But then $F$ is the direct sum of a finite number of copies of $K$, and is not an $I$-module by Lemma 6 . Similarly each $T_{p}$ is the direct sum of a finite number of modules of type $p^{\infty}$, and is not an $I$-module by Lemma 7 . It follows from Lemma 3 and Lemma 4 that $M$ is not an $I$-module.

Our problem is now limited to the consideration of reduced modules $M$. The torsion-free case is disposed of easily.

Theorem 3. A torsion-free R-module $M$ which is not divisible is an $I$. module.

Proof. If $M$ is not divisible, there exists $\alpha \neq 0$ in $R$ and $m \in M$ such that $\alpha x=m$ has no solution in $M$. Then the mapping $y \rightarrow \alpha y, y \in M$ is an isomorphism of $M$ onto a proper submodule.

Before considering torsion reduced modules, we obtain a final theorem concerning modules of finite rank.

THEOREM 4. Let $M$ be an R-module of finite rank. Then $M$ is an I-module if and only if $M / T$, where $T$ is the torsion submodule of $M$, is not divisible.

Proof. Suppose first that $M / T$ is divisible. Then $M / T$, being a torsionfree divisible module of finite rank, is not an $I$-module by Lemma 6 . Also, by Theorem 1, $T$ is not an $I$-module. Selecting $M(0)=0, M(1)=T$, and $M(2)$ $=M$, the hypotheses of Baer's Theorem are satisfied, and hence $M$ is not an $I$-module.

Conversely, if $M / T$ is not divisible, then there exists a prime $p \in R$ such that $p(M / T) \subset M / T$, and $\phi: x \rightarrow p x$ is an isomorphism of $M / T$ onto the proper submodule $p(M / T)$. Since $M$ has finite rank, each $T_{p}$ has finite rank. Hence $T_{p}$ is the direct sum of a module of bounded order and a divisible module [7, p. 53], and therefore is a direct summand of $M[5$, p. 18], $M$ $=T_{p} \oplus M^{\prime}$. Now we show that the mapping $\theta: y \rightarrow p y$ is an isomorphism of $M^{\prime}$ onto a proper submodule of $M^{\prime}$, so that by Lemma $1, M$ is an $I$-module. Let 
$T=T_{p} \oplus T^{\prime}$. Then the torsion submodule of $M^{\prime}$ is $T^{\prime}$, and $p y=0$ for $y \in M^{\prime}$ implies $y \in T_{p} \cap T^{\prime}=0$. Hence $\theta$ is an isomorphism. Since $p(M / T) \subset M / T$, and since $M^{\prime} / T^{\prime}$ is isomorphic to $M / T$, there exists an element $z \in M^{\prime}$ which is not divisible by $p$ modulo $T^{\prime}$. Hence $z \notin p M^{\prime}$, so that $\theta$ is not onto $M^{\prime}$. This completes the proof.

We now let $M$ be a reduced torsion module. We may assume that $R$ is not a field, since $M=0$ is the only torsion module over a field. By Lemma 4, we may assume that $M$ is primary.

Let $\boldsymbol{\aleph}$ be a cardinal number. Then we will say that $M$ is $\boldsymbol{\aleph}$-generated if $M$ has a generating set with $\boldsymbol{\aleph}$ elements and $\boldsymbol{\aleph}$ is the least cardinal with this property. If $M$ is $\boldsymbol{\aleph}$-generated, we will write $|M|=\boldsymbol{\aleph}$. We denote the cardinal number of a set $S$ by $\|S\|$.

Theorem 5. Let $M$ be a p-primary reduced R-module. If $|M|>\|R\| \boldsymbol{\aleph}_{0}$, then $M$ is an I-module.

Proof. Since $R$ is not a field, $\|R\| \geqq \boldsymbol{\aleph}_{0}$. Thus, by hypothesis, $|M|>2 \aleph_{0}$. It follows that $|B| \geqq \boldsymbol{N}_{0}$, where $B$ is a basic submodule (see [6, p. 181]) of $M$. For if $|B|<\boldsymbol{N}_{0}, B$ is finitely generated, which implies that $B$ is a pure submodule of $M$ of bounded order and is therefore a direct summand of $M$. Thus $M=B \oplus B^{\prime}$, where $B^{\prime}$ is divisible, but since $M$ is reduced, $B^{\prime}=0$. Then $M$ is finitely generated, which is a contradiction. Following Fuchs $[3, \mathrm{p}$. 273-274], we obtain

$$
|M| \leqq \boldsymbol{\aleph}_{0}\|B\| \boldsymbol{\aleph}_{0}+|B|=\|B\| \boldsymbol{\aleph}_{0},
$$

since $|B| \leqq\|B\|$ and $|B| \geqq \boldsymbol{\aleph}_{0}$. Now $\|B\| \leqq\|R\| \cdot|B|$, so that

$$
|M| \leqq(\|R\| \cdot|B|) \aleph_{0} \text {. }
$$

Now assume that $|B|=\boldsymbol{\aleph}_{0}$. Then since $\|R\| \geqq \boldsymbol{\aleph}_{0},\|R\| \cdot|B|=\|R\|$ and $|M| \leqq\|R\| \boldsymbol{\aleph}_{0}$, which contradicts the hypothesis. Hence $|B|>\boldsymbol{\aleph}_{0}$, that is, $B$ is not countably generated. Since $B=B_{1} \oplus B_{2} \oplus \cdots \oplus B_{i} \oplus \cdots$ where $B_{i}$ is a direct sum of cyclic modules of order $p^{i}$, some $B_{j}$ is the direct sum of infinitely many cyclic modules of order $p^{j}$. But $B_{j}$ is a pure submodule of $M$ of bounded order and is a direct summand of $M$. Thus $M$ is an $I$-module by Lemma 1 and Lemma 2.

COROLlary. Let $G \neq 0$ be a reduced abelian group with torsion subgroup $T$. Then if either $\|T\|=1$ or $\|T\|>2 \aleph_{0}, G$ has an isomorphic proper subgroup.

Proof. If $\|T\|=1, G$ is torsion-free, and the result follows from Theorem 3. If $\|T\|>2 \aleph_{0}$, then for some primary component $T_{p}$ of $T,\left\|T_{p}\right\|>2 \aleph_{0}$. By the proof of Theorem 5, a component $B_{j}$ of a basic subgroup of $T_{p}$ is an $I$-group. Since $B_{j}$ is pure in $B, B$ is pure in $T_{p}, T_{p}$ is pure in $T$, and $T$ is pure in $G$, we have that $B_{j}$ is a pure subgroup of $G$ of bounded order, and is a direct summand of $G$. Thus $G$ is an $I$-group. 
It will follow from the results of the next section that if $M$ is a primary reduced module such that $|M|=\boldsymbol{\aleph}_{0}$, then $M$ is an $I$-module.

III. Partly transitive modules. Throughout this section, $M$ is a $p$-primary module over a principal ideal domain $R$. For the sake of completeness, we do not assume that $M$ is reduced, although for the application to $I$-modules it would be sufficient to consider the reduced case. We use the following standard notation of [5].

(1) $D$ is the maximal divisible submodule of $M$.

(2) $P=\{x \in M \mid p x=0\}$.

(3) $M_{\alpha}$, for any ordinal $\alpha$, is defined inductively as follows: (i) $M_{0}=M$, (ii) $M_{\alpha+1}=p M_{\alpha}$, (iii) $M_{\alpha}=\bigcap_{\beta<\alpha} M_{\beta}$ if $\alpha$ is a limit ordinal.

(4) $P_{\alpha}=P \cap M_{\alpha}$.

(5) $\lambda=\min \left\{\alpha \mid M_{\alpha}=M_{\alpha+1}\right\}$. It is clear that $M_{\lambda}=D$.

(6) $f(\alpha)=\operatorname{dim}\left(P_{\alpha} / P_{\alpha+1}\right)$, where $P_{\alpha} / P_{\alpha+1}$ is regarded as a vector space over $R /(p)$, for $\alpha<\lambda$. $f(\lambda)=\operatorname{dim}(D \cap P)=\operatorname{dim} P_{\lambda}$ over $R /(p) . f(\alpha)=0$ for $\alpha>\lambda$ (including $\alpha=\infty$ ).

(7) An element $x \in M, x \in D$, has height $\alpha$ if $x \in M_{\alpha}$ and $x \in M_{\alpha+1}$, and we write $h(x)=\alpha$. For $x \neq 0$ in $D$, we write $h(x)=\lambda$, and $h(0)=\infty$, where $\infty$ is greater than any ordinal.

(8) For $x \in M, U(x)=\left(h(x), h(p x), \cdots, h\left(p^{n} x\right), \cdots\right)$ is the Ulm sequence of $x$.

(9) $\mu=\min \left\{\gamma \mid \sum_{\gamma \leqq \alpha \leqq \lambda} f(\alpha)<\boldsymbol{\aleph}_{0}\right\}$, provided $f(\lambda)<\boldsymbol{N}_{0}$, and $\mu=\infty$ otherwise.

(10) $H=M_{\mu}$ if $\mu \leqq \lambda$, and $H=0$ if $\mu=\infty$.

We call $H$ the top submodule of $M$.

It should be remarked that for a $p$-primary module $M, M$ has finite rank $r$ if and only if $M \cap P$ has finite dimension $r$ over $R /(p)$. Suppose first that $M$ has finite rank $r$. Then by the result of Prufer [7], $M$ is the direct sum of $r$ modules each cyclic or of type $p^{\infty}$. Hence $M \cap P$ is the direct sum of $r$ cyclic modules of order $p$, which is equivalent to the statement that $M \cap P$ has dimension $r$ over $R /(p)$. Conversely, if $M \cap P$ has finite dimension $r$ over $R /(p)$, then the rank of $M$ cannot be less than $r$ by what we have shown above. Also, every finitely generated submodule $N$ of $M$ has rank equal to the dimension of $N \cap P$. Since $N \cap P \subseteq M \cap P$, the dimension of $N \cap P$ is not greater than $r$. Hence the rank of $M$ is not greater than $r$.

THEOREM 6. (a) If $\mu \leqq \lambda$, then $\lambda=\mu+n$ for some non-negative integer $n$. (b) $H$ has finite rank. (c) If $\theta$ is an isomorphism of $M$ into itself, then $\theta$ restricted to $H$ is an automorphism and $h(\theta x)=h(x)$ for all $x \in H$.

Proof. (a) We may suppose $\mu<\lambda$. If there were a limit ordinal $\alpha$ such that $\mu<\alpha \leqq \lambda$, then there would be infinitely many distinct ordinals $\gamma$ such that $\mu<\gamma<\alpha$ and $f(\gamma) \neq 0$. For let $Q$ be the set of ordinals $\gamma$ such that $\mu<\gamma<\alpha$ and $f(\gamma) \neq 0$. If $Q$ is empty, let $\beta=\mu$, and if $Q$ is not empty and finite, let $\beta$ be the 
greatest ordinal in $Q$ such that $f(\beta) \neq 0$. Then $0=f(\beta+1)=f(\beta+2)=\cdots$ for the infinite sequence of ordinals $\beta+1, \beta+2, \ldots$ which are less than $\alpha$. This implies that $P_{\beta+1}=P_{\beta+2}=\cdots$. Thus for $x \in P_{\beta+1}=M_{\beta+1} \cap P, x \in P_{\beta+n+1}$ $=M_{\beta+n+1} \cap P \subseteq p^{n} M_{\beta+1}$, so that $x$ is divisible by $p^{n}$ in $M_{\beta+1}$ for arbitrary $n>0$. Thus every $x \in P_{\beta+1}$ has infinite height in $M_{\beta+1}$, and this implies that $M_{\beta+1}$ is divisible by [5, Lemma 8]. But this is impossible since $M_{\beta+1} \supset M_{\lambda}=D$, the maximal divisible submodule of $M$. Now we have $\sum_{\mu \leq \gamma \leq \lambda} f(\gamma) \geqq \boldsymbol{\aleph}_{0}$, which contradicts the definition of $\mu$. Hence, $\lambda=\mu+n$ for some positive integer $n$.

(b) If $\mu=\infty, H=0$ has finite rank zero. Otherwise $\mu \leqq \lambda$. Then by (a), $\lambda=\mu+n$ for some non-negative integer $n$, and we prove by induction that $M_{\lambda-k}$ for $k \leqq n$ has finite rank. For $k=0, f(\lambda)$ is just the rank of $M_{\lambda}=D$, and $f(\lambda)$ is finite. Assume that $M_{\lambda-k+1}$ has finite rank. This means $P_{\lambda-k+1}$ is finite dimensional over $R /(p)$. But since the dimension of $P_{\lambda-k} / P_{\lambda-k+1}$ is $f(\lambda-k)$, which is finite, $P_{\lambda-k}$ has finite dimension. Since, as we have remarked above, the dimension of $P_{\lambda-k}$ is the rank of $M_{\lambda-k}$, the induction is complete.

(c) If $H=0$, there is nothing to prove, so that we can assume $\mu \leqq \lambda$. Then by (a) $\lambda=\mu+n$ for some non-negative integer $n$. Since $M_{\alpha}$ is fully invariant for all $\alpha, \theta\left(M_{\lambda-k}\right) \subseteq M_{\lambda-k}$ for $k=0,1,2, \cdots, n$. Since $\theta$ is an isomorphism, it follows from (b) and Theorem 1 that $\theta\left(M_{\lambda-k}\right)=M_{\lambda-k}$. This implies that $h(\theta x)=h(x)$ for all $x \in H$, and in particular that $\theta(H)=\theta\left(M_{\lambda-n}\right)=M_{\lambda-n}=H$, so that $\theta$ restricted to $H$ is an automorphism. This completes the proof of the theorem.

Let $x \in M$ and $\theta$ be an isomorphism of $M$ into itself. Let $U(x)$ $=\left(\alpha_{0}, \alpha_{1}, \cdots, \alpha_{r}, \infty, \infty, \cdots\right)$ and $U(\theta x)=\left(\beta_{0}, \beta_{1}, \cdots, \beta_{s}, \infty, \infty, \cdots\right)$. Then $\alpha_{i} \leqq \beta_{i}$ for all $i$. It follows from Theorem 6 that if $\beta_{i} \geqq \mu$, then $\alpha_{i}=\beta_{i}$. This fact is the basis of the following definitions.

Definition 3. Let $U(x)=\left(\alpha_{0}, \alpha_{1}, \cdots, \alpha_{r}, \infty, \infty, \cdots\right)$ and $U(y)$ $=\left(\beta_{0}, \beta_{1}, \cdots, \beta_{s}, \infty, \infty, \cdots\right)$ be the Ulm sequences of elements $x, y \in M$. Then we write $U(x) \prec U(y)$ if $\alpha_{i} \leqq \beta_{i}$ for all $i$ and $\alpha_{i}=\beta_{i}$ whenever $\beta_{i} \geqq \mu$.

Definition 4. A $p$-primary $R$-module $M$ is called partly transitive if whenever $x, y \in M$ satisfy $U(x) \prec U(y)$, then there exists an isomorphism $\theta$ of $M$ into itself such that $\theta(x)=y$.

Theorem 7. A countably generated p-primary R-module $M$ is partly transitive.

Proof. Step 1. There is an isomorphism $\theta$ of $\{H, x\}$ onto $\{H, y\}$ which does not decrease height, where $x, y \in M$ satisfy $U(x) \prec U(y)$.

We note first that since $H$ has finite rank by Theorem $6(\mathrm{~b}), H$ is countably generated, and hence is transitive [5, Theorem 24]. Let $U(x)$ $=\left(\alpha_{0}, \alpha_{1}, \cdots, \alpha_{r}, \infty, \infty, \cdots\right), U(y)=\left(\beta_{0}, \beta_{1}, \cdots, \beta_{s}, \infty, \infty, \cdots\right)$, and let $k$ be the least integer such that $\beta_{k} \geqq \mu$. Since $U(x)<U(y)$, then for $j \geqq k, \alpha_{j}=\beta_{j}$, so that $U\left(p^{k} x\right)=U\left(p^{k} y\right)$. Moreover $h\left(p^{k-1} x\right) \leqq h\left(p^{k-1} y\right)<\mu$ for $k \geqq 1$. Now $p^{k} x$, 
$p^{k} y \in H$, and since $H$ is transitive, there exists an automorphism $\alpha$ of $H$ such that $\alpha\left(p^{k} x\right)=p^{k} y$. For $a \in H$ and $r \in R$, define $\theta:\{H, x\} \rightarrow\{H, y\}$ by $\theta(a+r x)$ $=\alpha(a)+r y$.

(i) $\theta$ is well-defined. For $a+r x=b+s x$ implies $(r-s) x \in H$, so that $r-s$ $=l p^{k}$. Then $\alpha(b)-\alpha(a)=\alpha(b-a)=\alpha\left(l p^{k} x\right)=l \alpha\left(p^{k} x\right)=l p^{k} y=r y-s y$. Hence $\theta(a+r x)=\alpha(a)+r y=\alpha(b)+s y=\theta(b+s x)$.

(ii) $\theta$ is clearly a homomorphism of $\{H, x\}$ onto $\{H, y\}$.

(iii) $\theta$ is $(1-1)$. Suppose $\theta(a+r x)=0$, that is, $\alpha(a)+r y=0$. Then $r=l p^{k}$ for otherwise $h(r y)<\mu$, in which case $\infty=h(\alpha(a)+r y)=h(r y)<\mu$, which is impossible. Thus $\alpha(r x)=\alpha\left(l^{k} x\right)=l \alpha\left(p^{k} x\right)=l p^{k} y=r y$, so that $0=\alpha(a)+r y$ $=\alpha(a)+\alpha(r x)=\alpha(a+r x)$. Since $\alpha$ is an automorphism, $a+r x=0$.

(iv) $\theta$ does not decrease height. Let $a+r x \in\{H, x\}$. If $p^{k}$ does not divide $r, h(\theta(a+r x))=h(\alpha(a)+r y)=h(r y) \geqq h(r x)=h(a+r x)$. If $p^{k}$ does not divide $r$, $a+r x \in H$, so that $\theta(a+r x)=\alpha(a+r x)$. Since $\alpha$ is an automorphism of $I I=M_{\mu}$, and since $M_{\mu+1}, M_{\mu+2}, \cdots, M_{\mu+n}=M_{\lambda}$ are fully invariant submodules of $H$ of finite rank, it follows from Theorem 1 that $\alpha\left(M_{\mu+k}\right)=M_{\mu+k}$, that is, $\alpha$ preserves $M$-height in $H$. Hence $h(\theta(a+r x))=h(\alpha(a+r x))=h(a+r x)$.

Step 2. Let $z_{1}, z_{2}, \cdots, z_{n}, \cdots$ be an enumeration of the generators of $M$, and let $x, y \in M$ with $U(x) \prec U(y)$. Assume that an isomorphism $\theta$ has been defined on a submodule $S$ of $M$ such that

(i) $S \supseteq\left\{H, x, z_{1}, \cdots, z_{n}\right\}, z_{n+1} \notin S$.

(ii) $S$ has finite rank.

(iii) $\theta(S) \subseteq M$ with $\theta(x)=y$.

(iv) $h(\theta s) \geqq h(s)$ for all $s \in S$.

Let $S^{\prime}=\left\{S, z_{n+1}\right\}$. We will extend $\theta$ to $S^{\prime}$, preserving the properties (iii) and (iv). We may assume that $p z_{n+1} \in S$, for we can replace the given sequence of generators by $p^{n_{1}} z_{1}, p^{n_{1}-1} z_{1}, \cdots, p z_{1}, z_{1}, p^{n_{2}} z_{2}, p^{n_{2}-1} z_{2}, \cdots, p z_{2}, z_{2}, \cdots$ where $p^{n_{i+1}} z_{i}=0$ for all $i$.

We prove first that there exists $z \in z_{n+1}+S$ which has maximal height in this coset, that is, $h(z) \geqq h(z+s)$ for all $s \in S$. By (ii), $S=\bar{S} \oplus E$, where $\bar{S}$ is finitely generated and $E \subseteq D$. This follows from the result in [7] referred to in the proof of Theorem 1 . If $\left(z_{n+1}+\bar{S}\right) \cap D$ is not empty, then there exists $z=z_{n+1}+s, s \in \bar{S}$ such that $h(z)=\lambda$. Then if $z^{\prime} \in z_{n+1}+S$ such that $h\left(z^{\prime}\right)>h(z)$, we have $h\left(z^{\prime}\right)=\infty$. Thus $z^{\prime}=0$ and $z_{n+1} \in S$, which is a contradiction. Hence $z$ has maximal height in $z_{n+1}+S$. Suppose, on the other hand, that $\left(z_{n+1}+\bar{S}\right) \cap D$ is empty. Then, since $\bar{S}$ is finitely generated, there exists $z=z_{n+1}+s, s \in \bar{S}$ such that $h(z)$ is maximal in $z_{n+1}+\bar{S}$. Let $z+s_{1}+t_{1}, s_{1} \in \bar{S}, t_{1} \in D$, be an element of $z_{n+1}+S$. Now $z+s_{1} \in D$, since $z+s_{1}=z_{n+1}+\left(s+s_{1}\right) \in z_{n+1}+\bar{S}$ and $\left(z_{n+1}+\bar{S}\right) \cap D$ is empty. Hence $h\left(z+s_{1}\right)<\lambda=h\left(t_{1}\right)$, so that $h(z) \geqq h\left(z+s_{1}\right)$ $=h\left(z+s_{1}+t_{1}\right)$. Thus $h(z)$ is maximal in $z_{n+1}+S$.

Let $T=\theta(S), w=\theta(p z)$, where $z$ is an element of maximal height in $z_{n+1}+S$. We prove next that there exists $z^{\prime} \in M$ such that (a) $p z^{\prime}=w$, (b) $h\left(z^{\prime}\right) \geqq h(z)$, and (c) $z^{\prime} \notin T$. 
We have $h(w)=h(\theta p z) \geqq h(p z) \geqq h(z)+1$, so that there exists $u \in M$ such that $h(u) \geqq h(z)$ and $w=p u$. If $u \notin T$, let $z^{\prime}=u$. Otherwise, $u \in T$, and let $\gamma=h(z)$. Since $z_{n+1} \notin H$ by (i), $z \notin H$, and $\gamma<\mu$. Therefore $\sum_{\gamma \leq \alpha \leq \lambda} f(\alpha)$ is infinite, and $\operatorname{dim}_{R /(p)} P_{\gamma}=\boldsymbol{\aleph}_{0}$. Since $S$ has finite rank by (ii), $T=\theta(S)$ has finite rank and $\operatorname{dim}_{R /(p)}(T \cap P)<\boldsymbol{\aleph}_{0}$. Thus there exists $v \in M$ such that $h(v) \geqq \gamma, p v=0$, and $v \notin T$. Set $z^{\prime}=u+v$. Then $z^{\prime} \notin T$, for otherwise $v=z^{\prime}-u$ $\in T$, and

$$
h\left(z^{\prime}\right) \geqq \min \{h(u), h(v)\} \geqq \gamma=h(z) .
$$

As $p z^{\prime}=w, z^{\prime}$ has properties (a), (b), and (c).

For $s \in S, r \in R$, define $\theta(s+r z)=\theta(s)+r z^{\prime}$. We show that this definition extends $\theta$ to $S^{\prime}$, preserving the properties (iii) and (iv). If $s+r z=s_{1}+r_{1} z$, then $\left(r-r_{1}\right) z=s_{1}-s \in S$. Since $z \equiv z_{n+1} \bmod S,\left(r-r_{1}\right) z_{n+1} \in S$. But $z_{n+1} \in S, p z_{n+1} \in S$, and the fact that $R$ is a principal ideal domain, imply $p$ divides $r-r_{1}$. Then $s_{1}-s=p m z$, and $\theta\left(s_{1}\right)-\theta(s)=m \theta(p z)=m w=m p z^{\prime}=r z^{\prime}-r_{1} z^{\prime}$. Hence $\theta$ is well defined. Clearly, $\theta$ is a homomorphism. Suppose that $\theta(s)+r z^{\prime}=0$. Then $r=p m$. For otherwise, there exists $r_{1} \in R$ such that $r_{1} r \equiv 1 \bmod \left(\right.$ order $\left.z^{\prime}\right)$, and $0=r_{1} \theta(s)+r_{1} r z^{\prime}=\theta\left(r_{1} s\right)+z^{\prime}$. Hence $z^{\prime} \in T$, which contradicts (c). Hence $\theta$ is an isomorphism of $S^{\prime}$ into $M$ such that $\theta(x)=y$.

It remains to prove (iv) is satisfied for $S^{\prime}$, that is, $h(\theta(s+r z))=h\left(\theta(s)+r z^{\prime}\right)$ $\geqq h(s+r z)$. If $r=p m$, then $\theta(s)+r z^{\prime}=\theta(s)+p m z^{\prime}=\theta(s)+m w=\theta(s)+m \theta(p z)$ $=\theta(s+m p z)=\theta(s+r z)$, so that $h\left(\theta(s)+r z^{\prime}\right) \geqq h(s+r z)$, since (iv) is satisfied for $S$. If $p$ does not divide $r$, there exists $r_{1} \in R$ such that $r_{1} r \equiv 1 \bmod (\operatorname{order} z)$. Then $h(s+r z)=h\left(r_{1}(s+r z)\right)=h\left(r_{1} s+z\right) \geqq \min \left\{h\left(r_{1} s\right), h(z)\right\}$. On the other hand, since $z$ has maximal height in $z+S, h\left(r_{1} s+z\right) \leqq h(z)$. Thus, in case $h(z)$ $=h\left(r_{1} s\right)$ or in case $h(z) \neq h\left(r_{1} s\right)$, we have $h(s+r z)=\min \left\{h\left(r_{1} s\right), h(z)\right\}$. But now, $h(z) \leqq h\left(z^{\prime}\right)$ by (b) and $h\left(r_{1} s\right) \leqq h\left(\theta\left(r_{1} s\right)\right)$ by (iv) which is satisfied for $S$. Hence $h(s+r z) \leqq \min \left\{h\left(\theta\left(r_{1} s\right), h\left(z^{\prime}\right)\right\} \leqq h\left(\theta\left(r_{1} s\right)+z^{\prime}\right)=h\left(\theta\left(r_{1} s+z\right)\right)=h\left(r_{1} \theta(s+r z)\right)\right.$ $=h(\theta(s+r z))$.

The proof of the theorem is completed, by inductively extending $\theta$ to an isomorphism of $M$ into itself.

CoRollary. If $M / D$ is countably generated, then the p-primary $R$-module $M$ is partly transitive.

Proof. $M=M_{1} \oplus \sum_{\tau \in T} \oplus D_{\tau}$, where each $D_{\tau}$ is a module of type $p^{\infty}$. If $T$ is countable, then $M$ is countably generated and the result follows from the theorem. Otherwise, for $x, y \in M$, there is a finite subset $R \subset T$ such that $x, y \in M_{1} \oplus \sum_{\tau \in R} \oplus D_{r}$. Let $S$ be a countably infinite set with $R \subset S \subset T$. Define $D_{1}=\sum_{\tau \in S} \oplus D_{\tau}$. Then $M_{1} \oplus D_{1}$ is a direct summand of $M$ which is countably generated, contains $x$ and $y$, and its top submodule is zero. Now if $U(x) \prec U(y)$ in $M$, we have $U(x) \prec U(y)$ in $M_{1} \oplus D_{1}$, so that by the theorem, there exists an isomorphism $\theta$ of $M_{1} \oplus D_{1}$ into itself such that $\theta(x)=y$, and, clearly, this isomorphism can be extended to $M$. 
Theorem 8. If $M$ is a p-primary reduced module such that $|M|=\boldsymbol{\aleph}_{0}$ then $M$ is an I-module.

Proof. If $\mu \geqq \omega$, then by the argument used to prove part (a) of Theorem 6 , there exist infinitely many $\alpha<\mu$ with $f(\alpha) \neq 0$. In particular, there exist $x, y \in M$ such that $p x=p y=0$ and $h(x)<h(y)<\mu$. Hence $U(x) \prec U(y)$, and by Theorem 7 , there is an isomorphism of $M$ into itself sending $x$ into $y$. Since this isomorphism is not height preserving, it cannot be an automorphism. Hence $M$ is an $I$-module.

If $\mu<\omega$, then $\lambda<\omega$ by Theorem 6 . Hence $M$ has bounded order $p^{\lambda}$. Therefore $M=M_{1} \oplus M_{2} \oplus \cdots \oplus M_{\lambda}$, where $M_{i}$ is the direct sum of cyclic modules of order $p^{i}$. Since $M$ is not finitely generated, some $M_{i}$ must have infinitely many summands and hence is an $I$-module by Lemma 2 . Therefore, by Lemma $1, M$ is an $I$-module.

Corollary. A countable p-primary reduced abelian group $G$ has an isomorphic proper subgroup if and only if $G$ is infinite.

IV. Summary. In this section we summarize our results for abelian groups, which is the most important special case of the problem.

For torsion groups $G$, the problem of determining groups with isomorphic proper subgroups ( $I$-groups) has been reduced to the case where $G$ is an infinite $p$-primary reduced group, and we have proved that if $G$ is countable (Theorem 8, Corollary) or if $G$ has cardinality greater than $2^{\aleph_{0}}$ (Theorem 5, Corollary), then $G$ is an $I$-group. No example of an infinite $p$-primary reduced group which is not an $I$-group is known to the authors. Further, we know of no example of such a group which is not partly transitive. It seems reasonable to conjecture that every $p$-primary group is partly transitive, in which case the problem would be completely solved for torsion groups, since by the proof of Theorem 8 , every $p$-primary, reduced, partly transitive, infinite group is an $I$-group.

For mixed abelian groups $G$ (where we may again assume that $G$ is reduced), the problem of determining $I$-groups has been reduced to the torsion case if $G$ splits, that is, if $G=T \oplus F$ where $T$ is the torsion subgroup of $G$ (Lemma 3 and Theorem 3). Theorem 4 disposes of mixed groups of finite rank, and the Corollary of Theorem 5 solves the problem when the cardinality of $T$ is greater than $2^{\aleph_{0}}$. Thus, the problem is open for mixed abelian groups of infinite rank which do not split and for which the cardinality of $T$ is less than or equal to $2^{\aleph_{0}}$. For this case, we give below an example of an $I$-group and an example of a non- $I$-group. We conjecture that $G$ is an $I$ group if $G / T$ is not divisible.

EXAMple 1. We use the example [6, p. 203] of a group $G=\left\{T, v_{1}, v_{2}, \cdots\right.$, $\left.v_{i}, \cdots\right\}$ where $T$ is a reduced $p$-primary group containing elements of arbitrarily high order, $T$ is the torsion subgroup of $G$, and the $v_{i}$ satisfy relations $p v_{i+1}=v_{i}+a_{i}, i=1,2, \cdots, a_{i} \in T . G$ has infinite rank since $T$ has infinite 
rank [7, p. 38]. It is shown that it is possible to choose $a_{i} \in T$ so that $G$ does not split. Now $G / T$ is a torsion-free group of rank one and type [6, p. 204, footnote] $\left(0, \cdots, 0, \infty \infty^{p}, 0, \cdots\right)$. If $q$ is a prime different from $p$, then $q(G / T) \subset G / T$ and $q G \subset G$. Since $T$ is $p$-primary, it follows as in the proof of Theorem 4 that the mapping $x \rightarrow q x, x \in G$, is an isomorphism of $G$ onto the proper subgroup $q G$.

ExAmple 2. Let $G$ be the complete direct sum of the cyclic groups $\left\{x_{1}\right\},\left\{x_{2}\right\}, \cdots,\left\{x_{i}\right\}, \cdots$, where $\left\{x_{i}\right\}$ has order $p_{i}$ and $p_{1}, p_{2}, \cdots$, $p_{i}, \cdots$ are the primes in their natural order. Since $G$ is uncountable, $G$ has infinite rank. The torsion subgroup $T$ of $G$ is the direct sum $T=\sum_{i=1}^{\infty} \oplus\left\{x_{i}\right\}$. If $\theta$ is an isomorphism of $G$ into $G$, then $\theta\left(\sum_{i=1}^{\infty} n_{i} x_{i}\right)=\sum_{i=1}^{\infty} n_{i} \theta\left(x_{i}\right)$. We have

$$
\sum_{i=1}^{\infty} n_{i} x_{i}=\sum_{i \neq j} n_{i} x_{i}+n_{j} x_{j}
$$

and

$$
\theta\left(\sum_{i \neq j} n_{i} x_{i}+n_{j} x_{j}\right)=\theta\left(\sum_{i \neq j} n_{i} x_{i}\right)+n_{j} \theta\left(x_{j}\right) .
$$

Since $\sum_{i \neq j} n_{i} x_{i}$ is divisible by $p_{j}, \theta\left(\sum_{i \neq j} n_{i} x_{i}\right)$ is divisible by $p_{j}$, and this implies $\theta\left(\sum_{i \neq j} n_{i} x_{i}\right)=\sum_{i \neq j} m_{i} x_{i}$. Further, $\left\{x_{j}\right\}$ is a fully invariant subgroup of $G$, so that $n_{j} \theta\left(x_{j}\right)=m_{j} x_{j}$. Hence we have $\theta\left(\sum_{i=1}^{\infty} n_{i} x_{i}\right)=\sum_{i=1}^{\infty} m_{i} x_{i}$, where $m_{j} x_{j}=n_{j} \theta\left(x_{j}\right)$, and this result holds for all $j$. Now $\theta$ restricted to $\left\{x_{j}\right\}$ is an automorphism, so that $\theta$ is a mapping onto $G$ and hence is an automorphism. Thus, $G$ is not an $I$-group. It should be observed that in this example $G / T$ is divisible, and that the example may be given for any principal ideal domain with infinitely many primes.

\section{REFERENCES}

1. R. Baer, Groups without proper isomorphic quotient groups, Bull. Amer. Math. Soc. vol. 50 (1944) pp. 267-278.

2. R. A. Beaumont, Groups with isomorphic proper subgroups, Bull. Amer. Math. Soc. vol. 51 (1945) pp. 381-387.

3. L. Fuchs, On the structure of abelian p-groups, Acta Math. Acad. Sci. Hung. vol. 4 (1953) pp. $267-287$.

4. I. Kaplansky, A note on groups without isomorphic subgroups, Bull. Amer. Math. Soc. vol. 51 (1945) pp. 529-530.

5. - Infinite abelian groups, University of Michigan Publications in Mathematics, no. $2,1954$.

6. A. G. Kurosh, The theory of groups, vol. 1, New York, 1955.

7. H. Prufer, Untersuchungen über die Zerlegbarkeit der abzahlbaren primaren Abelschen Gruppen, Math. Z. vol. 17 (1923) pp. 35-61.

UNIVERSITY OF WASHINGTON, Seattle, Wash. 